ARTICLE

\title{
High-throughput automated microfluidic sample preparation for accurate microbial genomics
}

Soohong Kim¹,2, Joachim De Jonghe ${ }^{1,3}$, Anthony B. Kulesa1,2, David Feldman ${ }^{1,4}$, Tommi Vatanen ${ }^{1,5}$, Roby P. Bhattacharyya ${ }^{1,6}$, Brittany Berdy ${ }^{7}$, James Gomez ${ }^{1}$, Jill Nolan', Slava Epstein ${ }^{7}$ \& Paul C. Blainey ${ }^{1,2}$

Low-cost shotgun DNA sequencing is transforming the microbial sciences. Sequencing instruments are so effective that sample preparation is now the key limiting factor. Here, we introduce a microfluidic sample preparation platform that integrates the key steps in cells to sequence library sample preparation for up to 96 samples and reduces DNA input requirements 100 -fold while maintaining or improving data quality. The general-purpose microarchitecture we demonstrate supports workflows with arbitrary numbers of reaction and clean-up or capture steps. By reducing the sample quantity requirements, we enabled low-input ( $\sim 10,000$ cells) whole-genome shotgun (WGS) sequencing of Mycobacterium tuberculosis and soil micro-colonies with superior results. We also leveraged the enhanced throughput to sequence $\sim 400$ clinical Pseudomonas aeruginosa libraries and demonstrate excellent single-nucleotide polymorphism detection performance that explained phenotypically observed antibiotic resistance. Fully-integrated lab-on-chip sample preparation overcomes technical barriers to enable broader deployment of genomics across many basic research and translational applications.

\footnotetext{
${ }^{1}$ The Broad Institute of MIT and Harvard, Cambridge, Massachusetts 02142, USA. ${ }^{2}$ Department of Biological Engineering, Massachusetts Institute of Technology, Cambridge, Massachusetts 02139, USA. ${ }^{3}$ Department of Biochemistry, University of Cambridge, Cambridge CB2 1GA, UK. ${ }^{4}$ Department of Physics, Massachusetts Institute of Technology, Cambridge, Massachusetts 02139, USA. ${ }^{5}$ Department of Computer Science, Aalto University School of Science, Espoo 02150, Finland. ${ }^{6}$ Division of Infectious Diseases, Massachusetts General Hospital, Boston, Massachusetts 02114, USA. ${ }^{7}$ Department of Biology, Northeastern University, Boston, Massachusetts 02115, USA. Correspondence and requests for materials should be addressed to P.C.B. (email: pblainey@broadinstitute.org).
} 
ow-cost DNA sequence data generation is enabling the widespread application of genomic methods across the microbial sciences. Genome sequencing can comprehensively survey commensal microbiota ${ }^{1}$, enable the diagnosis of drug resistant infections ${ }^{2-6}$ and reveal networks through which infections are transmitted ${ }^{7}$. In particular, pathogen surveillance by whole-genome shotgun (WGS) analysis provides information for molecular epidemiology of critical value to public health ${ }^{7,8}$ that cannot be obtained by culture or PCR. To this point, a recent Executive Order ${ }^{9}$ called for nationwide tracking of antibiotic resistance in microbial pathogens by genome sequencing in the US. In addition, natural products produced by microbes continue to serve as a rich source of therapeutic compounds spanning antibiotics to cancer ${ }^{10}$. Such compounds can be discovered by performing large-scale sequencing of environmental samples ${ }^{11}$.

Despite impressive progress in technology for sequence data production, the methods used to prepare sequencing samples lag behind (Supplementary Fig. 1). To sequence bacterial genomes, cells must be lysed and their DNA purified, fragmented, tagged with adaptors and size-selected before loading on a sequencing instrument. The complex experimental logistics and labour currently required to complete these steps limit sample throughput. The introduction of liquid handling robotics and electrowettingbased 'digital' microfluidics have helped to increase throughput, but these workflows require high DNA input, do not integrate all the key workflow steps (variously omitting cell lysis, DNA fragmentation and size selection), and substantially offset reductions in reagent and labour costs with expensive proprietary equipment and consumables (Supplementary Fig. 2) ${ }^{12}$.

The performance of available sample preparation methods on low-quantity samples is limiting in many microbial applications, as microbes can be difficult to isolate and grow to the quantities required for sequencing. Data from samples that are expanded by extensive culture or biochemical amplification can be significantly biased and are more likely to be contaminated ${ }^{13-15}$. Available library construction techniques typically require inputs of $>1$ million cell equivalents and are susceptible to contamination (particularly at low-input levels) ${ }^{16-18}$.

Whole-genome sequencing holds promise as a low-cost, rapid, essentially universal diagnostic for infectious disease ${ }^{19-21}$. However, input quantity requirements present a serious barrier for rapid WGS-based diagnostics for slow-growing microbes like Mycobacterium tuberculosis. Applications in environmental microbiology and natural product discovery are even further restricted by high-input requirements since only about $1 \%$ of environmental microbial isolates are easily cultured to produce a large quantity of pure genomic material ${ }^{22}$. Innovations in environmental sampling and culturing such as the iChip $^{22}$ enhance the chance of producing novel isolates, but also produce micro-colonies that are recalcitrant to scale-up culture and provide too little biomass for available direct WGS approaches.

Here we introduce a new poly dimethylsulfoxide (PDMS) microfluidic circuit architecture that integrates all the major steps in sample preparation for the first time (Supplementary Fig. 2; Supplementary Data 1) and makes major advances in input requirement and throughput while maintaining data quality. We demonstrated the new microarchitecture for sample preparation from clinical pathogen and environmental isolates, showing excellent data quality at extraordinarily low-input quantities. We expect this sample preparation platform will find wide application in a variety of high sample throughput genomics applications ${ }^{2-5,23-26}$.

\section{Results}

Microdevice design and operation. Microfluidics is a natural solution to increase throughput and reduce input requirements thanks to its scalable automation and capability to precisely manipulate small volumes ${ }^{27,28}$. Even small bench-top sequencing instruments like the MiSeq can sequence many microbial genomes per run, necessitating larger sample preparation batch sizes. Thus, we optimized a high-density two-layer microfluidic ${ }^{27}$ sample-processing microarchitecture that enables a batch size up to 96 samples per device run (Fig. 1 and Supplementary Fig. 3). Integrating all the key WGS sample preparation steps in our automated microfluidic device resulted in increased throughput, reduced reagent consumption, reduced input requirements, reduced contamination and improved reproducibility.

To prepare samples for genome sequencing, DNA must first be extracted from cells and then processed into properly sized fragments with attached sequencing adaptors. We extract genomic DNA in the device by lysing cells with a combination of heat, detergents, and hydrolytic enzymes. To fragment and attach adaptors to the gDNA, we apply the 'tagmentation' chemistry $^{23-26,29}$, which uses transposase enzymes to insert adaptor oligonucleotide sequences directly into high molecular weight DNA. For DNA purification, which is required at multiple points in the sample preparation process, we implement the solid phase reversible immobilization $(S P R I)^{30}$ method in the microfluidic system (Supplementary Note 1).

As we increased the reactor density to fit 96 channels in a $70 \mathrm{~mm} \times 35 \mathrm{~mm}$ device, we adapted our design to prevent cross contamination among samples and reagents, while still allowing for a single shared waste stream and individual collection of products.

Our microfluidic circuit performs the DNA extraction and library construction steps in a rotary reactor ${ }^{31}$ of 36 nanoliters (nl) that metres and mixes reagents and operates in concert with filter valves to strain cells or beads from solution (Fig. 1a and Supplementary Fig. 4). Standard micro-valves partition the rotary reactor for dead-end-fill loading ${ }^{27}$ of precise quantities of each reagent. These same valves are used coordinately as peristaltic pumps to mix reaction components within the rotary reactors (Supplementary Fig. 3a). Filter valves are improved sieve valves ${ }^{27}$ that can be actuated to rapidly strain particles from solution (Supplementary Fig. 5). To concentrate cells or purify, concentrate, and size-select nucleic acid products, we capture beads using filter valves at the output of each reactor (Supplementary Fig. 3a). This purification approach requires no chemical modification of the device ${ }^{32}$ and simplifies the reaction circuit since the filter valves can be directed to collect or release beads by flow in either direction, eliminating the need for additional bypass channels that reduce reactor density ${ }^{33}$. The micro-automated SPRI purifications used in DNA extraction, reaction clean-up and size selection operations yield $>80 \%$ recovery of picogram-level starting material (Supplementary Fig. 3b).

To establish multiple manifolds for solution loading/unloading in the two-layer high-density format, we strategically placed waste port 'vias' within each filter unit that connect to a 'sewer line' in the bottom layer of the device ${ }^{33}$ (Supplementary Fig. 3a). This arrangement enables bead, sample and reagent wastes to escape the high-density reaction circuits in the device upper layer without cross contamination (Supplementary Fig. 6; Supplementary Note 2) and without the complexity of extra device layers or extra space needed for internal access ports ${ }^{34}$. We initially load reagents into each reactor through one manifold (red arrow, Fig. 1a), while cells or gDNA samples are loaded via individual input/output ports (black arrow, Fig. 1a).

Finally, the re-use of each rotary reactor for multiple process steps eliminates the need for a series of reactors matched to each workflow step. Re-use is enabled by the capability for reaction/pull-down (for example, filtration or purification) steps. 
a

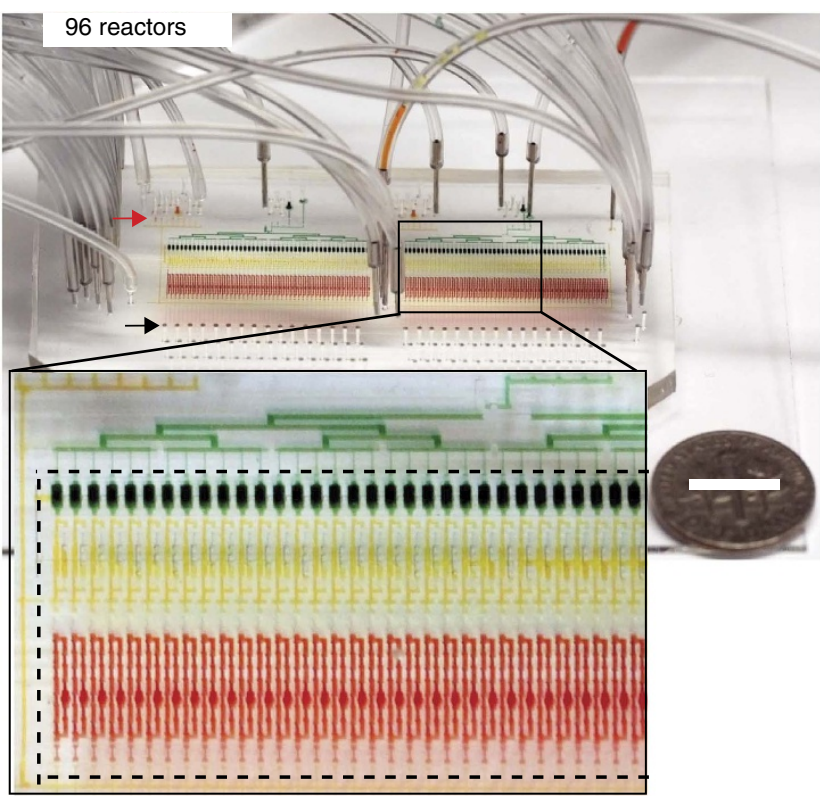

b

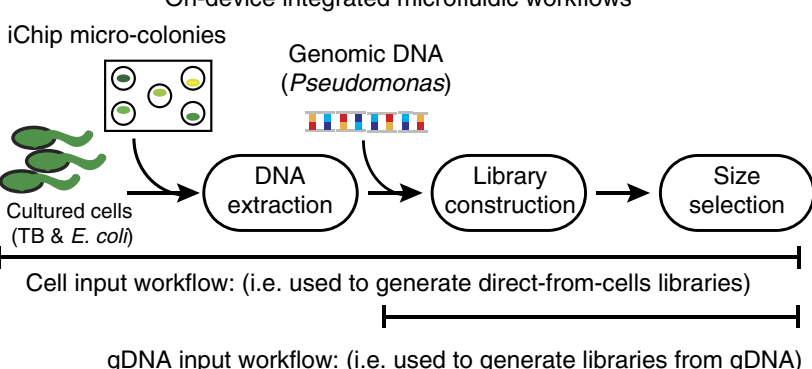

C

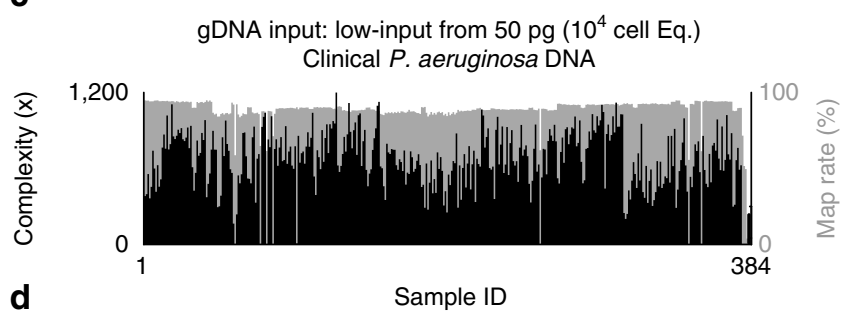

d
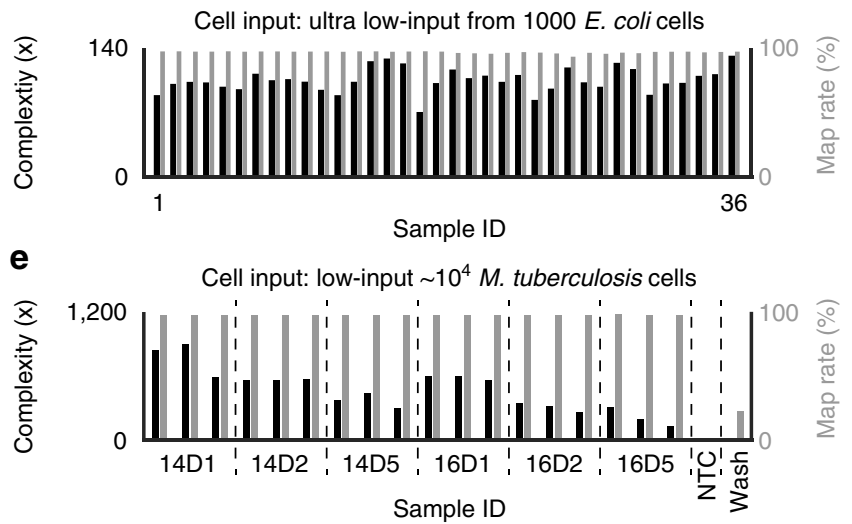

f

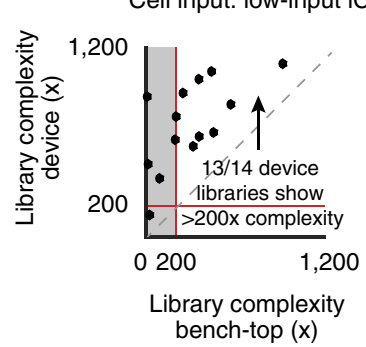

ip $\sim 10^{4}$ soil micro-colony cells

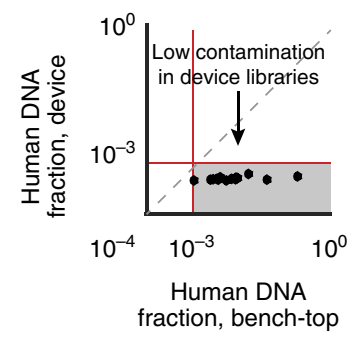

Figure 1 | Genomic sample preparation device operation and performance. (a) A photograph of the $96 \times 36 \mathrm{nl}$ microfluidic sample preparation device filled with food colouring to highlight features. Dime indicates scale (white bar indicates $1 \mathrm{~cm}$ ). Inset: the reactor (red), filter (yellow) and reservoir (green) units. Black and red arrows designate reagent input ports and sample input/output ports, respectively. (b) The microfluidic sample preparation workflows for biomass input (extreme left) and gDNA input (top). (c-e) Estimated sequence library complexity (units of fold-coverage) and mapping rate for (c) the clinical $P$. aeruginosa isolate gDNA samples mapped to PA-14 (of the 384 samples, eight individual replicates were lost completely during the barcoding step, leaving two replicates for each of these isolates. We know that these PCR drop-outs occurred due to faulty primers because these particular eight primer sets were subsequently observed to fail consistently across multiple samples). (d) Low-input E. coli biomass samples mapped to BL21-DE3 and (e) low-input M. tuberculosis biomass samples mapped to OFXR-14. (f) Comparison of microfluidic and optimized bench-top sample preparation from low-input from soil micro-colony biomass (left, library complexity; right, human contamination). The library complexities were calculated using Picard tools (http:// broadinstitute.github.io/picard/) and the human DNA read fraction was determined using deconseq (http://deconseq.sourceforge.net/).

The combination of reactor re-use for an arbitrary number of process steps together with pull-down/purification capability makes the system largely protocol-independent since most next-generation sequencing (NGS) and cell-handling processes can be accomplished with some number of these elementary steps in combination.

Low-input DNA extraction and library construction. The extraction and library construction workflow uses each reactor for five different reaction steps (lysis, $2 \times$ DNA precipitation, tagmentation and reaction stop) and the filter unit for three different capture steps (cell capture and $2 \times$ SPRI bead capture). Low sample input capability requires high efficiency lysis, purification and tagmentation, as well as contamination resistance to prevent small samples from being overwhelmed by extraneous DNA. We validated our microfluidic platform by processing four different low-input samples; samples of $\sim 1,000$ E. coli cells, $\sim 10,000$ M. tuberculosis cells, $\sim 10,000$ cell soil micro-colony samples, and GC-rich genomic DNA samples from clinical $P$. aeruginosa isolates (Supplementary Data 2).

The microfluidic devices consistently converted $5-15 \%$ of input gDNA into library molecules as estimated by qPCR measurement of output tagmentation products (Supplementary Fig. 3c). This value is significantly higher than previously reported low-input library construction efficiencies of $0.5-2 \%$ (ref. 35). Efficiency estimates derived from the duplicate sequence read rate agreed with our qPCR analysis ${ }^{36}$ (Supplementary Note 3). Libraries constructed on-chip from 50-100 picograms gDNA input showed reproducible mapping rates, fragment sizes and insert sizes, and k-mer frequencies consistent with standard high-input sample preparation (Fig. 1c, Supplementary Figs $3 \mathrm{~d}$ and 7$)^{37}$.

On-device cell lysis and DNA extraction steps were also found to be highly efficient. We measured $10 \%$ end-to-end conversion efficiency and found good library quality when using an ultra-low 
input of just 1,000 E. coli cells, representing sample and reagent inputs 200 times lower than standard 'low input' protocols that specify $1 \mathrm{ng}$ input (Fig. 1d; Supplementary Note 3). High efficiency was also achieved for organisms like M. tuberculosis (10,000 cell input) and environmental micro-colonies that are known to be more challenging to lyse (Fig. 1e,f; Supplementary Note 4).

To test how contamination and sequence library quality compare at low input to conventional sample preparation, we carried out a matched comparison of environmental microcolony sequence libraries prepared by the microfluidic method with those prepared on the bench-top by an optimized low-input procedure using the same reagents in the same laboratory environment by the same operator (Supplementary Note 5).

For this comparison, we used soil samples from a private Boston garden grown using the iChip $^{22}$ system, which cultures environmental microbes in situ to produce micro-colonies (Supplementary Fig. 8). We estimated the iChip micro-colonies to contain on the order of 100,000 cells each in total, and the input for sample preparation to be on the order of 10,000 cells (Supplementary Note 4). Each sample was split in half. One part was directly loaded and processed in our device (Fig. $1 \mathrm{~b}$ and Supplementary Fig. 9), while the remaining portion was processed using an equivalent sample-processing technique at the bench-top. Comparing data from the two sample preparation methods produced from the same micro-colonies in the same HiSeq 2500 sequencing run, the on-device sample preparation yielded an average of twice the library complexity, half the coefficient of variation in complexity, no drop-outs and far lower human DNA contamination (Fig. 1f).

High-throughput library construction from clinical isolates. To test the potential for our approach to address the sampleprocessing bottleneck that limits application of microbial WGS analysis, we applied our system to process libraries from $124 P$. aeruginosa clinical isolates obtained from six randomly selected subjects from Brigham and Women's Hospital (Boston, MA). A total of 12 to $24 P$. aeruginosa colonies were isolated from each subject sample (Fig. 2a; Supplementary Data 3). To empirically determine the single-nucleotide polymorphism (SNP) false detection rate, colonies from two of the six subjects (P03 and P04) were each sampled from 'control' plates representing expansions of individual primary colonies. We extracted genomic DNA from each culture, normalized the DNA concentrations and loaded the samples into our microfluidic device for library construction. To enable analysis of any errors that occurred during sample preparation, sequencing or analysis ${ }^{38}$, we prepared triplicate technical replicate libraries from each DNA sample (Supplementary Note 6). A single pool of 384 barcoded $P$. aeruginosa and control libraries was sequenced across two HiSeq 2500 lanes (Supplementary Note 7 and 8).

Low-input libraries support high-specification SNP calling. In order to determine the genomic diversity across isolates and reliably detect sequence variants with important functional consequences like antibiotic resistance, sequencing libraries must not introduce systematic errors and maintain uniform genomic coverage. To compare our microfluidic Pseudomonas libraries to those produced using bench-top methods, we instantiated an informatics pipeline for SNP calling (Supplementary Fig. 10) with an allelic fraction (AF) threshold as the primary adjustable parameter controlling sensitivity and specificity (Supplementary Fig. 11). We define the AF as the fraction of quality-adjusted ${ }^{39}$ variant base counts at a given reference position. A lower AF threshold increases SNP calling sensitivity but increases false- positive calls arising from errors that might occur in library construction, sequencing and read mapping operations. We found that setting the AF threshold to 0.82 limited the number of discordant SNPs detected across replicate libraries to zero (using discordant SNPs as a heuristic for erroneous variant calls $^{38}$; Fig. 2b,c). To benchmark our parameterized pipeline, we analysed data from a previous study of SNPs in clinical Burkholderia dolosa isolates ${ }^{40}$ and successfully detected all the reported SNPs (Supplementary Data 4).

To validate the actual sensitivity and specificity of SNP calls in analysis of low-input microfluidic sequence libraries versus bench-top libraries, we prepared several libraries from the same isolate sample: three low-input (50 pg) microfluidic libraries and three high-input $(24 \mathrm{ng})$ bench-top libraries, each sequenced to $50 \times$ coverage; and an additional high-input 'gold standard' bench-top library sequenced to $340 \times$ coverage. At $50 \times$ mean coverage of the $50 \mathrm{pg}$ microfluidic libraries, no false-positive SNPs were detected versus the $340 \times$ high-input library, indicating that accuracy was better than $1.4 \times 10^{-7}$ when calling more than $99 \%$ of reference positions. At equal mean coverage, there was no significant decrease in the quality of SNP calls made from the picogram-input libraries produced in our device versus the nanogram-input bench-top libraries (Fig. 2d). In fact, the $50 \times$ low-input consensus base calling accuracy from the $50 \mathrm{pg}$ input samples compares favourably to that reported for advanced single-strand consensus sequencing approaches that depend on extraordinarily deep sequence coverage and extra sample preparation steps $\left(10^{-5}\right.$; ref. 41$)$. Although we expect replicate library sequencing to suppress rare sample preparation artifacts like PCR errors, our comparison revealed no significant difference in the accuracy of replicate library sequencing compared with single-library sequencing at equivalent depth in our samples (Fig. 2d), indicating that artifacts from library construction affected variant calling at a frequency below $10^{-7}$.

Comparative genomics of clinical Pseudomonas isolates. In examining sequence variation between isolates, we identified 25,000-90,000 SNP sites across isolates from different subjects (Fig. 2e), but isolates from the same subject were essentially clonal (Fig. 2f; Supplementary Data 5). We detected no SNP sites among three subjects' isolates (P01, P02 and P06) and one of the control plates (P03). Among isolates from subject P05, just two SNP sites were identified, and only one SNP site was identified among isolates from control plate P04, with 2 of 24 isolates showing evidence for the same variant at this locus (Supplementary Note 9). Because the variant base in these two samples was reproducibly detected across the technical replicates in both affected subject P04 isolates (six sequence libraries in total), we can exclude sample preparation and sequencing errors as explanations for the variant calls. Rather, these calls likely arose from a mixture of strains being transferred to the control plate from overlapping colonies on the primary plate or a mutation that occurred during subsequent laboratory culture.

Our antibiotic resistance phenotype testing and gene content analysis likewise showed homogeneity across isolates from a given subject and extensive differences between isolates from different subjects (Fig. 3a and Supplementary Fig. 12; Supplementary Note 10). The core genes that were common in isolates between subjects were enriched for function in central dogma processes, while genes that varied between subjects were enriched for functions related to DNA transposition, recombination, restriction-modification, and transition metal response genes tied to mercury resistance (Supplementary Fig. 13; Supplementary Data 6 and 7; Supplementary Note 11). To fully validate the small number of variant calls made among the patient-specific isolate 


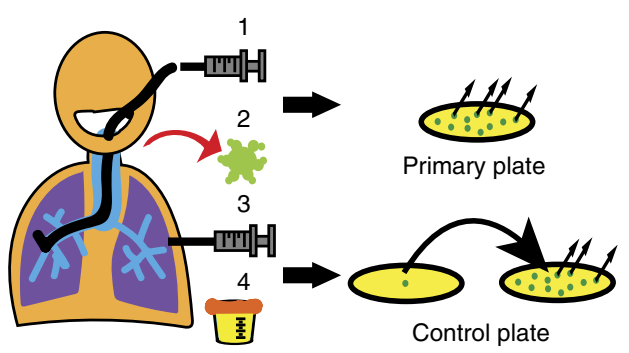

Concordant SNP:

Reference: A G C T A G C T

Rep1: $\quad$ AGT CAGCT

Rep2: $\quad A G T C A G C T$

Rep3: $A G T C A G C T$

Discordant SNP:

Reference: AGCTAGCT

Rep1: $\quad A G T C A G C T$

Rep2: $\quad A G T C A G C T$

Rep3: $\quad A G T T A G C T$

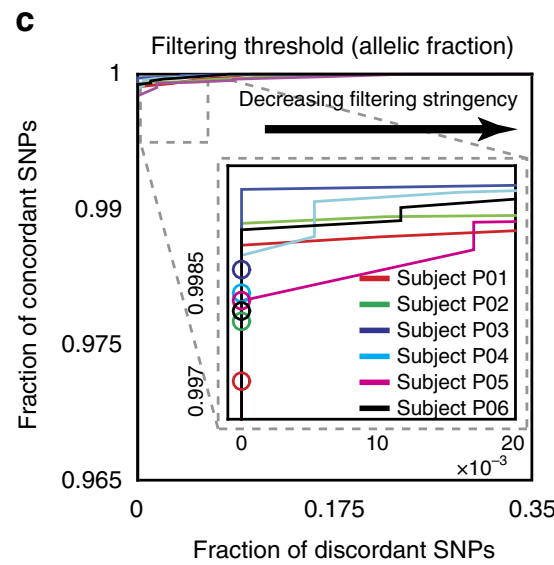

d
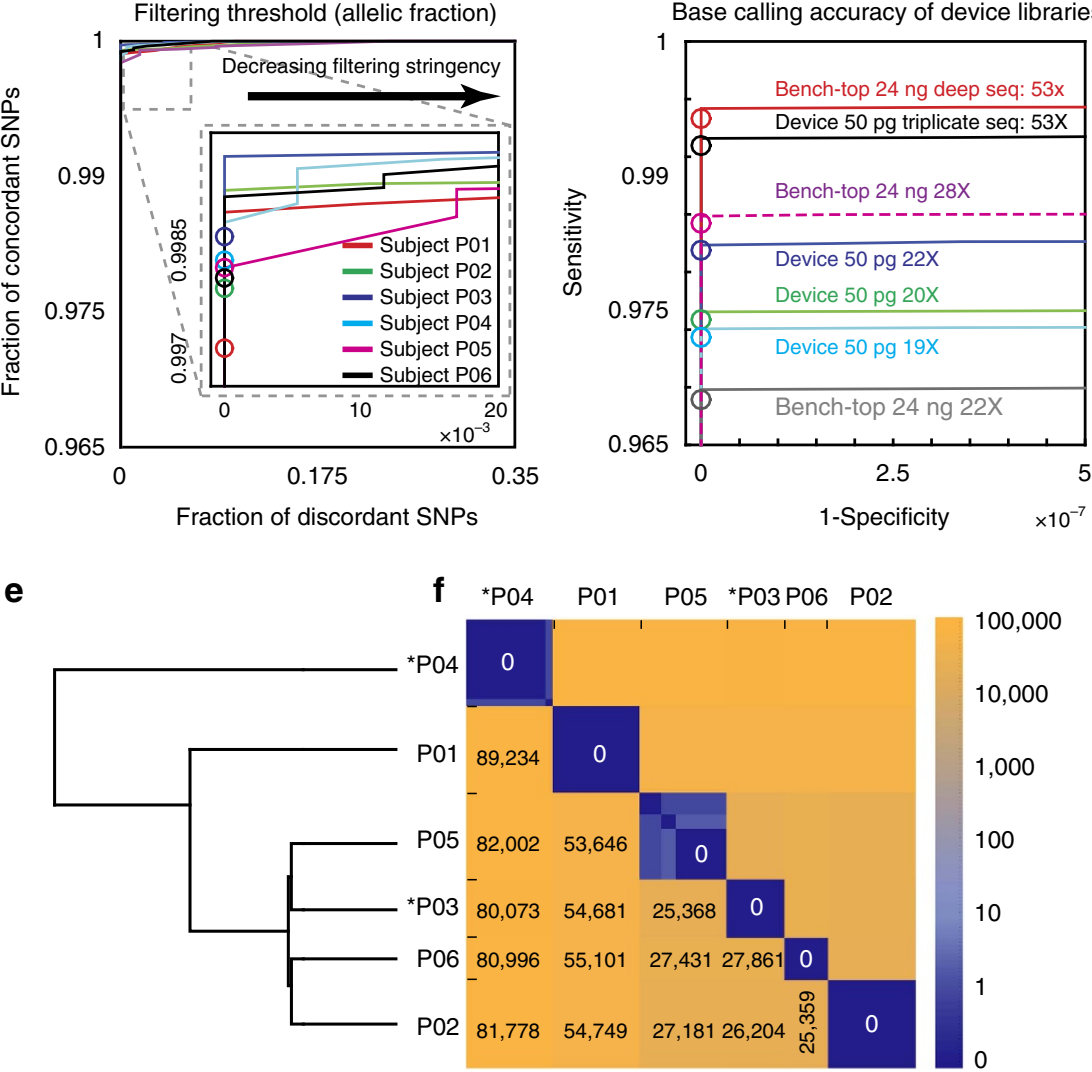

Figure 2 | Pseudomonas aeruginosa clinical isolate sequencing and error correction. (a) Four types of samples were collected from six different subjects; (1) bronchial alveolar lavage, (2) sputum, (3) thoracostomy and (4) urine. Samples were streaked on primary plates and two control plates, which are expanded from single colonies. (b) Concordant SNP call sets are defined as call sets that agree among all technical replicate libraries. (c) The fraction of concordant and discordant SNPs among technical replicates $(n=3)$ of one isolate from each subject (P01-4, P02-26, P03-58, P04-72, P05-105 and P06115 , where the notation P01-4 means patient subject 1, sample 4) is plotted with decreasing AF stringency (minimum read depth fixed at 6; minimum mapping quality fixed at 45; excluding indels; see 'Methods' section). Inset: thresholding at an AF value of 0.82 (circles) balances the maximization of concordant SNPs and minimization of discordant SNPs and was used for variant calling across all our samples. (d) Receiver operator characteristic (ROC) plot compares the accuracy and sensitivity in SNP calling among low-input libraries of sample P01-4 made in the device, on the bench-top libraries from individual replicates, from pooled triplicate replicates, and at different average coverage levels. With the AF threshold value at 0.82 (red circles), there is no meaningful difference between microfluidic and bench-top libraries, or between single replicate and pooled triplicates at equal coverage. Due to differences in gene content and genomic structure of the reference and the subject P01 strain, 7\% of the reference genome had no coverage in this analysis and 1.9\% of the remaining sequence was masked due to poor average read mapping quality $(\mathrm{MQ}<45$; Supplementary Note 9). (e) Homology tree constructed based on the number of inter-subject SNPs. (f) Heat map of SNPs between each sample, grouped by the patient subject. Asterisks indicate control plate isolates that were expanded from single colonies. Numbers represent median number of SNPs in each subject pair block.

populations, the no-calls we made at loci with metrics near our analysis thresholds (Supplementary Fig. 14; Supplementary Data 5; Supplementary Note 9), and the variants responsible for drug resistance (described below), we performed Sanger sequencing, finding agreement with the short-read analysis in all cases.
WGS sequencing accurately predicts antibiotic resistance. Our sequence analysis of the clinical Pseudomonas isolates identified variant bases known to confer resistance to imipenem, ciprofloxacin and ceftazidime, three antibiotics commonly used to treat pulmonary P. aeruginosa infections (Fig. 3a; Supplementary Data 8). All 24 isolates from subjects P01 and P04 had a 
a

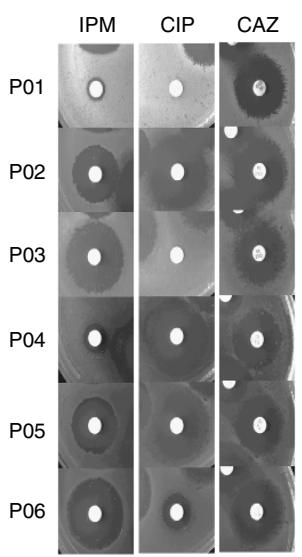

Disc diffusion antibiotic susceptibility test

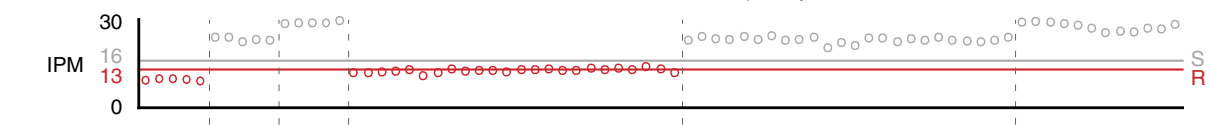

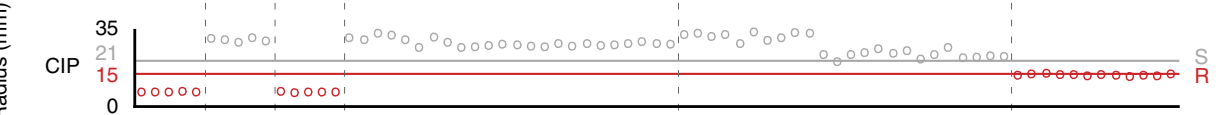

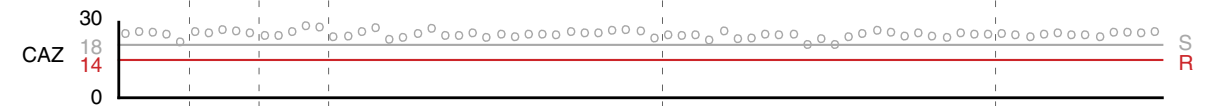

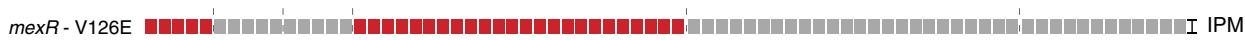

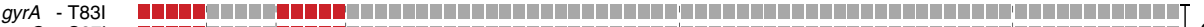

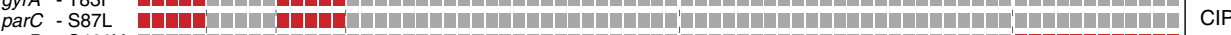

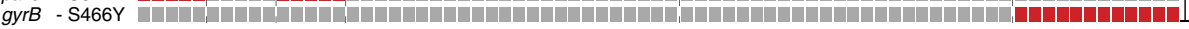
$\begin{array}{lllll}\text { P01 P02 }{ }^{\prime} \mathrm{P} 03{ }^{\prime} & \text { P04 } & \text { P05 } & \text { P06 }\end{array}$

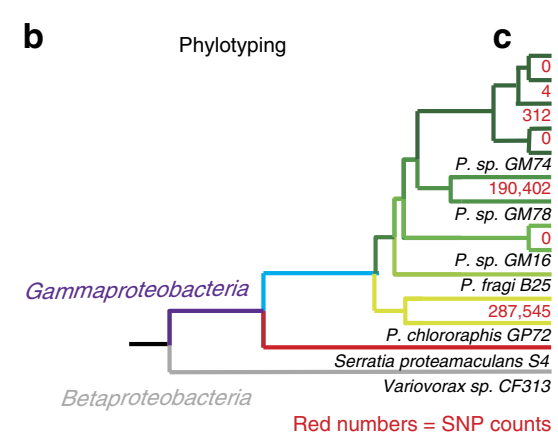

AntiSMASH analysis

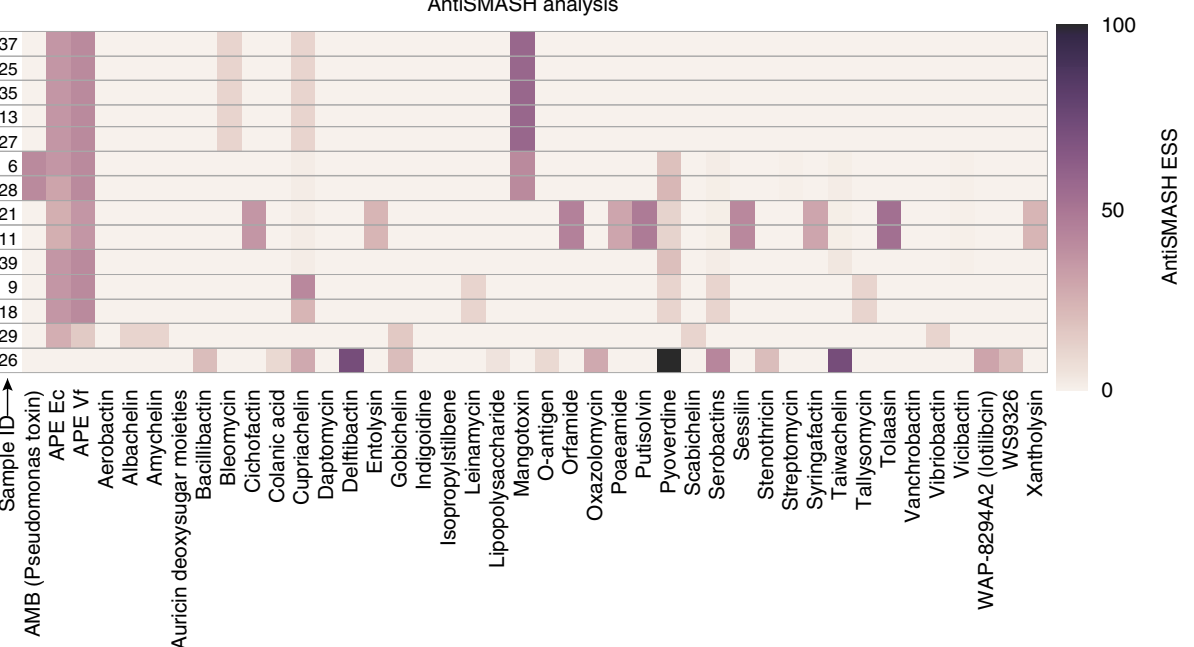

Figure 3 | Functional genomics from low-input microbial samples. (a) Antibiotic susceptibility phenotyping and genotyping. Antibiotic susceptibility was tested on a randomly sampled subset of isolates from each subject by the disc diffusion susceptibility assay. The drugs tested are IPM, CIP and CAZ (10 $\mu$ g imipenem, $5 \mu \mathrm{g}$ ciprofloxacin and $30 \mu \mathrm{g}$ ceftazidime, respectively). Raw images of one plate from each subject that was analysed (left). Plot shows inhibition zone radius for each antibiotic and known antibiotic resistance SNPs detected by WGS, grouped by subjects (right). The samples with smaller radii close to the Clinical \& Laboratory Standards Institute (CLSI) break point (red line) indicate samples that are resistant to the specific antibiotic (grey points). Radii greater than the green line indicates samples identified as susceptible by the CLSI break point (blue points). The first ten samples from subject P05 were measured on a separate day from the remaining P05 samples; the apparent difference in susceptibility in these samples and the remaining P05 samples is most likely due to a systematic difference in the assay (possibly image contrast) on the second day or degradation of the drug sample used between the two measurement sets (we classify all the P05 isolates tested as susceptible to ciprofloxacin). (b) Phylotyping of the soil micro-colonies and (c) secondary metabolite class prediction using AntiSMASH analysis of de novo assembled genomic contigs. The clustering of samples in the phylotyping results is

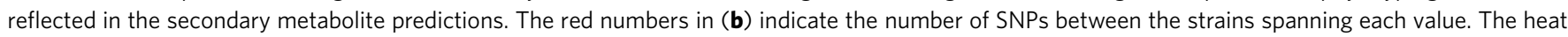
map values in (c) represent empirical similarity scores ${ }^{11}$.

non-synonymous SNP, V126E, in the mexR (efflux pump) gene consistent with previously reported imipenem resistance mutations ${ }^{42}$. Similarly, all isolates from subjects P01 and P03 had two SNPs, T83I in the gyrA (gyrase A) gene and S87L in the parC (topoisomerase IV) gene, each reported to yield ciprofloxacin resistance ${ }^{43}$. All 12 isolates from subject P06 had a ciprofloxacin resistance mutation, S466Y in the gyrB $^{44}$ (gyrase B) gene, that was not found in the other subjects. In our drug resistance phenotype test ${ }^{45}$ (Fig. 3a; Supplementary Note 10), 100\% of the phenotypically observed variability in resistance was explained by our low input, high-throughput WGS data (Fig. 3a).

Direct de novo WGS analysis of soil micro-colonies. Our microfluidic platform showed data performance equal to benchtop library construction methods with orders of magnitude less input, lending the confidence to carry out strain and natural product prediction from the iChip micro-colony samples. We de novo assembled the genome of each micro-colony data set produced as described above (Supplementary Data 9) and phylotyped the micro-colonies at the strain level using a multi-locus sequence typing analysis ${ }^{46}$.

Of the 14 colonies analysed, 12 represented five different known Pseudomonas strains ( $P$. $s p$ GM74, $P . s p$ GM78, $P . s p$ GM16, $P$. fragi B25 and P. chlororaphis GP72). We identified the remaining two colonies as Serratia proteamaculans S4 and Varivorax sp. CF313, respectively (Fig. 3b and Supplementary Fig. 15). Our high-GC Pseudomonas assemblies, based on lowinput sample-processing direct from cells, were more contiguous than recently reported draft environmental Pseudomonas assemblies produced from sequence libraries prepared by standard, high-input methods ${ }^{47}$ (Supplementary Data 9). The device was also more reliable in producing libraries from our micro-colony samples, as two of the bench-top libraries dropped 
out entirely during the sample preparation process (Fig. If and Supplementary Fig. 16) and six of the fourteen bench-top libraries were at or below a library complexity of 200 genomic equivalents, the minimum required to enable $50 \times$ unique coverage with acceptable read duplication rate.

Soil samples are frequently mined for natural products, recently yielding teixobactin, a promising agent under development for treating Gram-positive bacterial infections ${ }^{48}$. We analysed our micro-colony draft genomes for natural product genes using the anti-SMASH tool ${ }^{11}$ (Fig. 3c). While the metabolite-producing gene cluster profiles between the device libraries and bench-top libraries mostly agreed (except where the bench-top samples dropped out entirely), the Serratia and Varivorax samples gave divergent results, with the higherquality microfluidic libraries likely producing more accurate gene calls (Supplementary Fig. 16).

\section{Discussion}

Despite the ability of Illumina's HiSeq and NextSeq platforms to process hundreds of microbial sequence libraries per run, genomic analysis is underutilized in epidemiology, clinical care and natural product discovery due to the complexity, limited throughput, labour-intensity, and input quantity requirements of available sample preparation methods. Here, we piloted an automated microfluidic system that integrates at high throughput all the key steps in NGS sample preparation: cell concentration, lysis, fragmentation, adaptor tagging, fragment purification and size selection (Supplementary Fig. 2; Supplementary Data 2, 10 and 11). By lowering input requirements 200 -fold, our system also enables new applications such as rapid analysis of slow-growing pathogen isolates and direct analyses of samples with limited biomass.

We constructed nearly 400 WGS sequence libraries from 124 isolates of $P$. aeruginosa collected from six patients. Our analysis revealed tremendous inter-subject diversity in gene content and genome sequence even though all six samples were collected from the same hospital within a period of a few weeks. Despite the dynamic accessory genome known in Pseudomonas, we found negligible intra-subject variation in our samples, indicating that the infected sites contained essentially clonal Pseudomonas populations, not the diverse Pseudomonas populations often described in the chronic pulmonary infections common among cystic fibrosis patients (Supplementary Note 12). The population signature we observed is consistent with community-acquired infection or infection of these patients from their own microbiota in the hospital setting.

The genomic determinants of imipenem and ciprofloxacin resistance were apparent in our variant calls and concordant with phenotypic susceptibility assays. We also found substantial gene content and sequence variation across strains with the same multiple resistance phenotype, indicating that these strains most likely acquired resistance to the same set of drugs independently. As is often found in Pseudomonas, we noticed diversity in the gene content across the isolates from different patients, notably enrichment of DNA integration and restriction functions in the variable genome that may be related to horizontal gene transfer, subject-specific prophage (Supplementary Figs 17 and 18) and other putatively exogenous sequences (Supplementary Fig. 19; Supplementary Data 12; Supplementary Note 13), as well as mercury resistance elements, which have previously been linked to the development of antibiotic resistance ${ }^{49,50}$.

These analyses were enabled by the Q68 + SNP calling performance achieved by the tuned variant caller ${ }^{38}$. Replicate sequencing that enabled this performance increases the need for automation of sample preparation and low-input sample preparation, but has the power to discriminate sequencing errors from errors introduced during library construction including base damage, PCR-derived mutations, and chimeras.

We demonstrated that the microfluidic system's integrated DNA extraction capability works at very low-input levels for challenging sample types like $M$. tuberculosis cells and soil micro-colonies. This eliminates the need for pre-amplification by whole-genome amplification, which increases workflow complexity and degrades data quality. The input reduction to thousands of cells could cut diagnostic test times in half for slow-growing pathogens like $M$. tuberculosis. Lowering input requirements also enables new organism and natural product discovery by direct WGS analysis of environmental microbes that are hard to culture, such as the iChip micro-colonies. Side-by-side comparison of soil micro-colony WGS analysis with an optimized bench-top procedure makes clear the superior performance of the new microfluidic method in reliability and critical data metrics. Future synthetic biology approaches ${ }^{51}$ to synthesize natural product genes for expression in industrial production strains would place even higher demands on sequence and assembly quality. Re-discovery of known natural products is a limiting factor in the discovery of novel bioactive compounds ${ }^{52}$, placing a premium on isolating the most challenging-to-grow organisms that are enriched in diversity versus known microbes.

We expect the increased automation, throughput and low-input capability of our microfluidic library construction method to enable a wide variety of future applications. Current library preparation protocols require nanograms to micrograms of DNA that have been previously extracted from cells. Some protocols require that DNA be pre-fragmented and size-selected in addition. Eliminating such challenging input requirements could expand the use of less invasive but low-yield sampling and biopsy procedures, enable direct pathogen identification in tiny microbiome samples, better enrichment of cell types or tissue regions of interest in clinical micro-samples, and eliminate whole-genome pre-amplification from some workflows ${ }^{53}$. The high throughput and high accuracy sample preparation method presented here will power applications including precision medicine, genomic surveillance, antibiotic resistance tracking ${ }^{2-5}$ and novel organism/natural product discovery on large scales.

\section{Methods}

Microfluidic device fabrication. Microfluidic devices were fabricated by multilayer soft lithography of PDMS, a transparent silicone elastomer, on a mould comprised of a silicon wafer patterned with photoresist. Mould and device fabrication were carried out at the Broad Institute. Separate moulds were used to cast a control layer of height $50 \mu \mathrm{m}$ and a flow layer of height $6 \mathrm{~mm}$. The two layers were partially cured, aligned manually and thermally bonded by further curing. Inlet ports were punched and the two-layer PDMS device was bonded to a glass slide after activation by air plasma exposure.

The flow layer mould was patterned in five steps: (1) rectangular $15 \mu \mathrm{m}$, (2) rectangular $5 \mu \mathrm{m}$, (3) rounded $15 \mu \mathrm{m}$, (4) rectangular $30 \mu \mathrm{m}$ and (5) rectangular $100 \mu \mathrm{m}$ features. The rectangular features were made by spin coating SU-8 2001 $(5 \mu \mathrm{m}), 2015(15$ and $30 \mu \mathrm{m})$ and $2050(100 \mu \mathrm{m})$ photoresists (Microchem) on a silicon wafer. The coated wafers were patterned by ultraviolet exposure (OAI 206 mask aligner) through a mask printed at 20,000 dpi (Fineline; see Autocad design files for each layer in supplementary material), followed by feature development in SU-8 developer (Microchem). The rounded features were produced by spin coating AZ-4620 photoresist (Microchem) after coating with Hexamethyldisilazane (Sigma) and air-drying, patterning the wafer with UV exposure and a mask, developing in the AZ $400 \mathrm{~K}$ developer (Microchem), and slowly heating (from $65 \mathrm{C}$ to $190 \mathrm{C}$ over $4 \mathrm{~h}$ ) the wafer above the resist $\mathrm{Tg}(120 \mathrm{C})$ to round the features. The control layer mould was patterned in two steps: (1) rectangular $15 \mu \mathrm{m}$ and (2) rectangular $90 \mu \mathrm{m}$ features, using methods similar to those for the flow layer. Device production utilized standard soft lithography protocols. PDMS (Momentive) was mixed at 5:1 silicone to cross-linker ratio in a Thinky AR250 mixer, poured onto the flow layer mould, degassed in a vacuum chamber and cured by baking for $1 \mathrm{~h}$ at $80^{\circ} \mathrm{C}$. PDMS at a 20:1 silicone to cross-linker ratio was spin coated on the valve layer mould to a height of $50 \mu \mathrm{m}$, then baked at $80^{\circ} \mathrm{C}$ for $40 \mathrm{~min}$. The two layers were aligned under a stereomicroscope (Nikon), and further baked for $2 \mathrm{~h}$ at $80^{\circ} \mathrm{C}$ to complete thermal bonding. Inlet holes were punched into 
the two-layered PDMS device (Syneo, ID $660 \mu \mathrm{m}$ tips) on the designated input/output port features. The device was then exposed to atmospheric plasma for $30 \mathrm{~s}$ at a pressure of $1.3 \mathrm{mbar}$ (Diener ATTO), bonded to a clean glass slide and baked for $3 \mathrm{~h}$ at $80^{\circ} \mathrm{C}$.

Device design file and controller. Flow and control layers were pneumatically activated by an array of 40 solenoid valves (Pneumadyne) controlled by a USB interface (McMaster) connected to a computer. The device controller was homebuilt following designs and procedures specified by Dr Rafael Gómez-Sjöberg and the Prof. Stephen Quake group at Stanford University as described at: https://sites.google.com/site/rafaelsmicrofluidicspage/valve-controllers/usb-basedcontroller.

The valve operation pressure was 40 PSI and sample/reagent solutions were driven at 20 PSI.

The device CAD file is publicly available on: https://sourceforge.net/projects/ sk-dev-cad-analysis-software/files/Kim_supplementaryfiles.zip /download.

Clinical $\boldsymbol{P}$. aeruginosa isolate sample collection and culture. The clinical samples used in this study were discards from the Brigham and Women's Hospital microbiology lab, and were disconnected from patient meta-data. Samples from selected sites of infection were streaked on selective media plates containing MacConkey agar. 12 to $24 P$. aeruginosa colonies were identified by appearance and randomly picked (Fig. 2a). P. aeruginosa isolates were received at the Broad Institute as frozen liquid cultures. They were grown to mid-log phase at a concentration of $5 \times 10^{8}$ cells ml $^{-1}$ in lysogeny broth (LB). Culture density was monitored by OD600 using a UV-vis spectrophotometer.

DNA extraction from clinical isolates (bench-top method). DNA was purified from bacterial cultures using Qiagen DNeasy Blood and Tissue kits on the QIAcube instrument (Qiagen). The cell input was between one and two million cells per sample. After extraction, the concentration of purified gDNA was measured by absorption at $260 \mathrm{~nm}$ (Nanodrop) and normalized to $20 \mathrm{ng} \mu \mathrm{l}$.

M. tuberculosis culture. Two M. tuberculosis clinical isolates (OFXR14 and OFXR16) were grown in Middlebrook 7H9 medium supplemented with OADC (Becton Dickinson), $0.05 \%$ Tween 80 (Sigma) and $0.2 \%$ glycerol at $37 \mathrm{C}$ with shaking. Samples were taken at OD600 0.05 to 0.1 , which corresponds to $\sim 1.5-3 \times 10^{7}$ bacterial cells per ml. A $100 \mu$ l culture sample from each isolate was heat inactivated at $80^{\circ} \mathrm{C}$ for $2 \mathrm{~h}$, flash frozen and used for all analyses.

iChip soil bacteria culture. A $1 \mathrm{~g}$ sample of soil collected from a private garden in Boston, MA was agitated vigorously for $10 \mathrm{~min}$ in $10 \mathrm{ml}$ sterile phosphate buffered saline (PBS). The soil was left to settle for $5 \mathrm{~min}$ and the supernatant was diluted again in PBS. Dilutions were then mixed with molten agar media $(0.1 \mathrm{~g}$ starch, $1.0 \mathrm{~g}$ casamino acids, $15 \mathrm{~g}$ technical agar per 11 of $\mathrm{H}_{2} \mathrm{O}$ ). The soil suspension was diluted further with agar to achieve an average concentration of one cell per $100 \mu$. Then $100 \mu \mathrm{l}$ of the soil-agar suspension was dispensed into the each well of an iChip ${ }^{22}$, which had a $0.03 \mu \mathrm{m}$ polycarbonate membrane attached to the bottom via silicone glue. After the agar suspension was solid, a $0.03 \mu \mathrm{m}$ polycarbonate membrane was attached to the top of the iChip using silicone glue. The iChip was incubated in direct contact with moist soil in the dark for 2 weeks. After 2 weeks, iChips were disassembled and individual colonies were picked with sterile toothpicks using a dissection microscope, and placed into $15 \mu \mathrm{l}$ PBS for further analysis.

Tagmentation (bench-top method, $24 \mathrm{ng}$ input). Bench-top library construction was done following the Illumina Nextera protocol for tagmentation. $5 \mu \mathrm{l}$ of $4.8 \mathrm{ng} \mathrm{hl}^{-1}$ (24 ng total) of purified gDNA was mixed with $15 \mu \mathrm{l}$ of Nextera enzyme (Illumina), $5 \mu$ l of $5 \times$ Tagmentation buffer $(50 \mathrm{mM}$ Tris- $\mathrm{HCl} \mathrm{pH} 8.0$ (Sigma), $20 \mathrm{mM} \mathrm{MgCl} 2$ (Sigma)) and $10 \mu \mathrm{l}$ of $\mathrm{H}_{2} \mathrm{O}$, then incubated for $10 \mathrm{~min}$ at $58^{\circ} \mathrm{C}$. $2.5 \mu \mathrm{l}$ of stop solution ( $2.5 \%$ wt per vol SDS (Sigma) in $\mathrm{H}_{2} \mathrm{O}$ ) was added to the mixture, followed by incubation for $10 \mathrm{~min}$ at $72^{\circ} \mathrm{C}$. Tagmented DNA was purified by mixing with $49.5 \mu \mathrm{l}$ SPRI bead suspension (Beckman-Coulter), binding for $10 \mathrm{~min}$ at $25^{\circ} \mathrm{C}$, magnetically separating the beads, washing twice with ethanol and eluting the product off the beads with $50 \mu \mathrm{l}$ of $10 \mathrm{mM}$ Tris-HCl $\mathrm{pH}$ 8.0.

Commercial DNA extraction method (bench-top bead beating). We attempted using the MoBio Power-soil kit (Qiagen) with $7 \mu \mathrm{l}$ of cells $\left(\sim 10^{4}\right.$ total cells) to extract gDNA for library construction but did not obtain a sufficient quantity of tagmented DNA fragments after library construction $(<1$ picograms of amplifiable tagmented product; data not shown).

Library quantification (qPCR). Before enrichment PCR, an aliquot from each library produced on the device was quantified by qPCR to verify successful library construction. qPCR was performed by mixing $1 \mu \mathrm{l}$ of tagmented product with $0.5 \mu \mathrm{l}$ Eva green dye (Evrogen), $0.5 \mu \mathrm{l}$ of Rox reference dye (ROCHE), $0.5 \mu \mathrm{l}$ of N12 Nextera barcoding primer (Illumina), $0.5 \mu \mathrm{l}$ of E502 Nextera barcoding primer
(Illumina), $4 \mu \mathrm{l}$ of DNA polymerase ready mix (Illumina) and $3 \mu \mathrm{l}$ of $\mathrm{H}_{2} \mathrm{O}$, then performing $\mathrm{qPCR}$ in a real-time thermocycler (Stratagene MX3005p). The thermal programme was: $5 \mathrm{~min}$ at $72^{\circ} \mathrm{C}, 1 \mathrm{~min}$ at $95^{\circ} \mathrm{C}$, then 40 cycles of $(1) 10 \mathrm{~s}$ at $95^{\circ} \mathrm{C}$, (2) $30 \mathrm{~s}$ at $60^{\circ} \mathrm{C}$ and (3) $30 \mathrm{~s}$ at $72^{\circ} \mathrm{C}$. To quantify the properly adapted tagmented library molecules in each sample the qPCR amplification curve of each sample was compared with the curves resulting from analysis of purified standards (amplified, size-selected library) of similar average molecular weight and GC content. The reference libraries were quantified using the Qubit method (ThermoFisher) and Kapa library quantification kits (Kapa Biosystems).

Enrichment/barcoding PCR and sample pooling. The sequencing libraries created on the microfluidic devices were barcoded using the Broad Institute's dual barcoding primers (Broad Genomics Platform), for which validation was not yet complete. Eight libraries were lost in the enrichment PCR step from the Pseuodomonas set due to bad primers.

$4 \mu \mathrm{l}$ of each tagmented sample library $(\sim 5 \mathrm{pg})$ was mixed with $0.5 \mu \mathrm{l}$ of primer $1,0.5 \mu \mathrm{l}$ of primer $2,4 \mu \mathrm{l}$ of amplification mix (Illumina) and $1 \mu \mathrm{l}$ of $\mathrm{H}_{2} \mathrm{O}$. Our libraries were amplified by 15 cycles of PCR (optimized for $5 \mathrm{pg}$ input), SPRI purified and Qubit quantified. The libraries were then pooled into a single mix in equal concentrations based on their Qubit-measured concentrations.

DNA sequencing. Quality assessment runs (library construction efficiency, sensitivity versus specificity, k-mer abundance and fragment size distribution) and production runs of test libraries were carried out by sequencing across variably configured Illumina MiSeq runs $(2 \times 50,2 \times 75,2 \times 150,2 \times 250$ and $2 \times 300)$. The quality assessed samples were sequenced on the Illumina Hiseq 2500 platform $(2 \times 125$ cycles or $2 \times 101$ cycles $)$ in the Broad Genomics Platform.

Antibiotic susceptibility testing. Clinical Pseudomonas aeruginosa isolates were grown to mid-log phase in Luria broth (LB), and $100 \mu \mathrm{l}$ of each culture was spread on an LB agar plate and allowed to dry for $15 \mathrm{~min}$ face-up at room temperature. Disks impregnated with ceftazidime ( $30 \mu \mathrm{g}$, Becton Dickinson), ciprofloxacin $(5 \mu \mathrm{g}$ Becton Dickinson) or imipenem (10 $\mu \mathrm{g}$, Becton Dickinson) were placed at evenly spaced distances on these plates, which were then incubated for $16 \mathrm{~h}$ at $37^{\circ} \mathrm{C}$. Plates were imaged using a FluorChem FC2 system (Alpha-Innotech) set to the visible range, and the radius of the zone of inhibition around each disk was measured using a custom Matlab image analysis script. These radii were compared with standards determined by the Clinical Laboratory Standards Institute (CLSI). The breakpoints for each antibiotic are as follow ( $S$ and $R$ stand for susceptible and resistant, respectively):

(1) Ciprofloxacin: $\mathrm{S}>21 \mathrm{~mm} ; R<15 \mathrm{~mm}$

(2) Imipenem: $S>16 \mathrm{~mm} ; R<13 \mathrm{~mm}$

(3) Ceftazidime: $S>18 \mathrm{~mm} ; R<14 \mathrm{~mm}$

Analysis software and commands. Software and commands used for sequencing analysis are available in the supplementary methods. Custom analysis software is publicly available on https://sourceforge.net/projects/sk-dev-cad-analysis-software/ files/Kim_supplementaryfiles.zip/download.

Data availability. Primary accessions. National Center for Biotechnology Information (NCBI) Sequence Read Archive (SRA)

clinical $P$. aeruginosa raw reads fastq PRJNA295070

clinical $P$. aeruginosa de novo assemblies fasta PRJNA295070

low-input E. coli raw reads fastq PRJNA295070

M. tuberculosis raw reads fastq PRJNA295070

soil micro-colonies raw reads fastq PRJNA295070

Referenced accessions. NCBI Reference sequence

Pseudomonas aeruginosa UCBPP-PA14 complete genome NC_008463.1

Mycobacterium tuberculosis OFXR-14 scaffold GCF_000660185.1

Mycobacterium tuberculosis OFXR-16 scaffold GCF_000660225.1

\section{References}

1. Yatsunenko, T. et al. Human gut microbiome viewed across age and geography. Nature 486, 222-227 (2012).

2. Marvig, R. L., Sommer, L. M., Molin, S. \& Johansen, H. K. Convergent evolution and adaptation of Pseudomonas aeruginosa within patients with cystic fibrosis. Nat. Genet. 47, 57-64 (2014).

3. Dallman, T. J. et al. Whole-genome sequencing for national surveillance of shiga toxin-producing Escherichia coli O157. Clin. Infect. Dis. 61, 305-312 (2015).

4. Nasser, W. et al. Evolutionary pathway to increased virulence and epidemic group A Streptococcus disease derived from 3,615 genome sequences. Proc. Natl Acad. Sci. USA 111, E1768-E1776 (2014).

5. Chewapreecha, C. et al. Dense genomic sampling identifies highways of pneumococcal recombination. Nat. Genet. 46, 305-309 (2014). 
6. Smith, E. E. et al. Genetic adaptation by Pseudomonas aeruginosa to the airways of cystic fibrosis patients. Proc. Natl Acad. Sci. USA 103, 8487-8492 (2006).

7. Snitkin, E. S. et al. Tracking a hospital outbreak of carbapenem-resistant Klebsiella pneumoniae with whole-genome sequencing. Sci. Transl. Med. 4, 148 ral16 (2012).

8. Centers for Disease Control and Prevention Antibiotic-Resistant Gonorrhea STD information from CDC. Available at https://www.cdc.gov/std/gonorrhea/ arg/basic.htm.

9. Obama, B. Executive Order--Combating Antibiotic-Resistant Bacteria whitehouse.gov. the White house (2014). Available at https://www.whitehouse. gov/the-press-office/2014/09/18/executive-order-combating-antibiotic-resistantbacteria\&gt;

10. Newman, D. J. \& Cragg, G. M. Natural products as sources of new drugs over the 30 years from 1981 to 2010. J. Nat. Prod. 75, 311-335 (2012).

11. Weber, T. et al. antiSMASH 3.0--a comprehensive resource for the genome mining of biosynthetic gene clusters. Nucleic Acids Res. 43, W237-W243 (2015).

12. Kim, H. et al. A microfluidic DNA library preparation platform for next-generation sequencing. PLoS ONE 8, e68988 (2013).

13. de Bourcy, C. F. A. et al. A quantitative comparison of single-cell whole genome amplification methods. PLoS ONE 9, e105585 (2014).

14. Kram, K. E. \& Finkel, S. E. Rich medium composition affects Escherichia coli survival, glycation, and mutation frequency during long-term batch culture. Appl. Environ. Microbiol. 81, 4442-4450 (2015).

15. Blainey, P. C. The future is now: single-cell genomics of bacteria and archaea. FEMS Microbiol. Rev. 37, 407-427 (2013).

16. Salter, S. J. et al. Reagent and laboratory contamination can critically impact sequence-based microbiome analyses. BMC Biol. 12, 87 (2014).

17. Jones, M. B. et al. Library preparation methodology can influence genomic and functional predictions in human microbiome research. Proc. Natl Acad. Sci. USA 112, 14024-14029 (2015)

18. Brooks, J. P. et al. The truth about metagenomics: quantifying and counteracting bias in 16S rRNA studies. BMC Microbiol. 15, 66 (2015).

19. Witney, A. A. et al. Clinical use of whole genome sequencing for Mycobacterium tuberculosis. BMC Med. 14, 46 (2016)

20. Wilson, M. R. et al. Actionable diagnosis of neuroleptospirosis by next-generation sequencing. N. Engl. J. Med. 370, 2408-2417 (2014).

21. Naccache, S. N. et al. A cloud-compatible bioinformatics pipeline for ultrarapid pathogen identification from next-generation sequencing of clinical samples. Genome Res. 24, 1180-1192 (2014).

22. Nichols, D. et al. Use of ichip for high-throughput in situ cultivation of \&quot; uncultivable\&quot; microbial species. Appl. Environ. Microbiol. 76, 2445-2450 (2010).

23. Anderson, S. Shotgun DNA sequencing using cloned DNase I-generated fragments. Nucleic Acids Res. 9, 3015-3027 (1981)

24. Ramsköld, D. et al. Full-length mRNA-Seq from single-cell levels of RNA and individual circulating tumor cells. Nat. Biotechnol. 30, 777-782 (2012).

25. Buenrostro, J. D., Giresi, P. G., Zaba, L. C., Chang, H. Y. \& Greenleaf, W. J. Transposition of native chromatin for fast and sensitive epigenomic profiling of open chromatin, DNA-binding proteins and nucleosome position. Nat. Methods 10, 1213-1218 (2013).

26. Robertson, G. et al. Genome-wide profiles of STAT1 DNA association using chromatin immunoprecipitation and massively parallel sequencing. Nat. Methods 4, 651-657 (2007).

27. Melin, J. \& Quake, S. R. Microfluidic large-scale integration: the evolution of design rules for biological automation. Annu. Rev. Biophys. Biomol. Struct. 36, 213-231 (2007)

28. Morinishi, L. S. \& Blainey, P. Simple bulk readout of digital nucleic acid quantification assays. J. Vis. Exp. e52925 (2015)

29. Caruccio, N. Preparation of next-generation sequencing libraries using Nextera technology: simultaneous DNA fragmentation and adaptor tagging by in vitro transposition. Methods Mol. Biol. 733, 241-255 (2011).

30. DeAngelis, M. M., Wang, D. G. \& Hawkins, T. L. Solid-phase reversible immobilization for the isolation of PCR products. Nucleic Acids Res. 23, 4742-4743 (1995).

31. Kim, S. et al. High-throughput single-molecule optofluidic analysis. Nat. Methods 8, 242-245 (2011).

32. Bhattacharyya, A. \& Klapperich, C. M. Thermoplastic microfluidic device for on-chip purification of nucleic acids for disposable diagnostics. Anal. Chem. 78, 788-792 (2006).

33. Tan, S. J. et al. A microfluidic device for preparing next generation DNA sequencing libraries and for automating other laboratory protocols that require one or more column chromatography steps. PLoS ONE 8, e64084 (2013).

34. Balagaddé, F. K., You, L., Hansen, C. L., Arnold, F. H. \& Quake, S. R. Long-term monitoring of bacteria undergoing programmed population control in a microchemostat. Science 309, 137-140 (2005).

35. Parkinson, N. J. et al. Preparation of high-quality next-generation sequencing libraries from picogram quantities of target DNA. Genome Res. 22, 125-133 (2012)
36. Li, H. Mathematical Notes on SAMtools Algorithms. (2010). Available at https://www.broadinstitute.org/gatk/media/docs/Samtools.pdf.

37. Kelley, D. R., Schatz, M. C. \& Salzberg, S. L. Quake: quality-aware detection and correction of sequencing errors. Genome Biol. 11, R116 (2010).

38. Robasky, K., Lewis, N. E. \& Church, G. M. The role of replicates for error mitigation in next-generation sequencing. Nat. Rev. Genet. 15, 56-62 (2014).

39. Walker, B. J. et al. Pilon: an integrated tool for comprehensive microbial variant detection and genome assembly improvement. PLOS ONE 9, e112963 (2014).

40. Lieberman, T. D. et al. Genetic variation of a bacterial pathogen within individuals with cystic fibrosis provides a record of selective pressures. Nat. Genet. 46, 82-87 (2014).

41. Schmitt, M. W. et al. Detection of ultra-rare mutations by next-generation sequencing. Proc. Natl Acad. Sci. USA 109, 14508-14513 (2012).

42. Pai, H., Kim, J., Lee, J. H., Choe, K. W. \& Gotoh, N. Carbapenem resistance mechanisms in Pseudomonas aeruginosa clinical isolates. Antimicrob. Agents Chemother. 45, 480-484 (2001).

43. Lomholt, J. A. \& Kilian, M. Ciprofloxacin susceptibility of Pseudomonas aeruginosa isolates from keratitis. Br. J. Ophthalmol. 87, 1238-1240 (2003).

44. Bruchmann, S., Dötsch, A., Nouri, B., Chaberny, I. F. \& Häussler, S Quantitative contributions of target alteration and decreased drug accumulation to Pseudomonas aeruginosa fluoroquinolone resistance. Antimicrob. Agents Chemother. 57, 1361-1368 (2013).

45. Bauer, A. W., Kirby, W. M., Sherris, J. C. \& Turck, M. Antibiotic susceptibility testing by a standardized single disk method. Am. J. Clin. Pathol. 45, 493-496 (1966).

46. Darling, A. E. et al. PhyloSift: phylogenetic analysis of genomes and metagenomes. PeerJ 2, e243 (2014).

47. Winsor, G. L. et al. Enhanced annotations and features for comparing thousands of Pseudomonas genomes in the Pseudomonas genome database. Nucleic Acids Res. 44, D646-D653 (2015).

48. Ling, L. L. et al. A new antibiotic kills pathogens without detectable resistance. Nature 517, 455-459 (2015).

49. Skurnik, D. et al. Is exposure to mercury a driving force for the carriage of antibiotic resistance genes? J. Med. Microbiol. 59, 804-807 (2010).

50. Rowland, I. R., Robinson, R. D. \& Doherty, R. A. Effects of diet on mercury metabolism and excretion in mice given methylmercury: role of gut flora. Arch Environ. Health 39, 401-408 (1984).

51. Smanski, M. J. et al. Synthetic biology to access and expand nature's chemical diversity. Nat. Rev. Microbiol. 14, 135-149 (2016).

52. Baltz, R. H. Antimicrobials from actinomycetes: back to the future. Microbe Am. Soc. Microbiol. 2, 125 (2007).

53. Fitzsimons, M. S. et al. Nearly finished genomes produced using gel microdroplet culturing reveal substantial intraspecies genomic diversity within the human microbiome. Genome Res. 23, 878-888 (2013).

\section{Acknowledgements}

We thank Dr Yonatan Grad, Dr Deb Hung, Dr Ashlee Earl, Dr Christopher Desjardins, Dr Robert Lintner, Mr Bruce Walker, Mr Terrance Shea, Ms Sheila Fisher, Dr Niall Lennon, Dr Stacy Gabriel and members of the Blainey Lab for helpful discussions. The indexing barcodes were provided by the Broad Institute Genomics Platform. We also thank Dr Lynn Bry at Brigham and Women's Hospital regarding sample collection and helpful discussions. This work was supported by the Burroughs Welcome Fund via a Career Award at the Scientific Interface to PCB, Broad Institute Startup Funds and an Emerging Technologies Opportunity Program award from the Department of Energy Joint Genome Institute. S.K. was supported by the National Science Foundation Postdoctoral Fellowship in Biology 1308852.

\section{Author contributions}

S.K. and P.C.B. designed the microfluidic device and planned experiments. S.K. fabricated the device, built the experimental hardware, performed the necessary experiments and optimization of on-device sample preparation, and processed the micro-colony samples and $M$. tuberculosis samples into sequence libraries. S.K. and A.B.K. wrote the hardware control software. S.K. and J.D.J. processed the clinical isolates in the device. S.K., J.D.J. and J.N. performed the enrichment PCR on the bench. S.K., A.B.K. and D.F. performed SNP analysis. S.K., T.V. and J.D.J. performed the gene content analysis and the variable genome content analysis. S.K. and R.P.B. performed the Pseudomonas antibiotic resistance analysis. S.K. and D.F. analysed soil micro-colony sequences. B.B. and S.E. provided soil micro-colony samples from the iChip. J.G. provided M. tuberculosis samples. S.K., A.B.K., D.F. and P.C.B. wrote the manuscript.

\section{Additional information}

Supplementary Information accompanies this paper at http://www.nature.com/ naturecommunications

Competing financial interests: The Broad Institute and MIT may seek to file for intellectual property on and/or commercialize aspects of this work. J.D.J., A.B.K., D.F., T.V., R.P.B., B.B., J.G., J.N. and S.E. declare no competing interests. 
Reprints and permission information is available online at http://npg.nature.com/ reprintsandpermissions/

How to cite this article: Kim, S. et al. High-throughput automated microfluidic sample preparation for accurate microbial genomics. Nat. Commun. 8, 13919 doi: 10.1038/ ncomms13919 (2017).

Publisher's note: Springer Nature remains neutral with regard to jurisdictional claims in published maps and institutional affiliations. (c) (i) This work is licensed under a Creative Commons Attribution 4.0 International License. The images or other third party material in this article are included in the article's Creative Commons license, unless indicated otherwise in the credit line; if the material is not included under the Creative Commons license, users will need to obtain permission from the license holder to reproduce the material. To view a copy of this license, visit http://creativecommons.org/licenses/by/4.0/

(C) The Author(s) 2017 